\title{
Watch Television
}

National Cancer Institute

\section{Source}

National Cancer Institute. Watch Television. NCI Thesaurus. Code C98074.

The act of spending time viewing a television. 